\title{
Multi-layer Neural Network for Servo Motor Control
}

\author{
Lalithamma GA \\ Assistant Professor, Dept of E\&E \\ SJBIT, Mandya
}

\author{
P.S. Puttaswamy,Ph.D \\ Prof, Deptt of E\&E \\ PESCE, Mandya
}

\author{
Kashyap D Dhruve \\ Planet-I Technologies \\ Bangalore
}

\begin{abstract}
AC servo systems are extensively used in robotic actuators and are competing with DC servo motors for motion control because of their favorable electrical and mechanical properties. This paper presents an approach towards the control system tuning for the speed control of an AC servo motor. An approach towards speed control of servo motor in presence of system parameter variations is presented. Multi-layer Artificial Neural Networks are designed and trained to model the plant parameter variations. Improvements in the speed control performance are presented for smaller variations and larger variations in the motor parameters and the load conditions.
\end{abstract}

KEYwORDS-PID, AC servo motor, multi-layer ANN, direct torque control.

\section{INTRODUCTION}

The PID control algorithm is generally used to control almost all loops in the process industries, AC servomechanisms and drive applications. In order to use a controller, it must first be tuned to the system requirements. The tuning synchronizes the controller with the controlled variable, thus allowing the process to be kept at its desired operating condition. Standard methods for tuning controllers and criteria for judging the loop tuning have been used for many years. Among the available methods some of them are based on mathematical criteria such as Cohen- Coon method, some are trial and error method, such as Continuous cycling method, relay feedback method and Kappa-Tau tuning method. Existing methods even though they have some advantages they have some difficulties while defining the solution for tuning the PID controller. Pre-selection of a controller structure can pose a challenge in applying PID control to a system. As vendors often recommend their own design procedure for controller structures, the tuning rules adopted for a specific controller structure may not perform well with other controller structure [1]. Therefore, one solution is seen to provide support for individual structure is through software. Detailed discussions on the use of various PID structures are presented in [1]. Nonetheless, controller parameters are required to be tuned such that the closed-loop control system would be stable and meet the given objectives associated with the following functions

1. Stability robustness.

2. Set-point following and tracking performance at transient, including rise-time, overshoot, and settling time.

3. Regulation performance at steady-state including load disturbance rejection.

4. Robustness against plant modeling uncertainty.
5. Noise attenuation and robustness against environmental uncertainty.

In industrial process there are many systems having nonlinear properties. Moreover these properties are often unknown and time varying. The commonly used PID controllers are simpler to realize but they suffer from poor performance if there are uncertainties and nonlinearities in the system[2]. Therefore PID controllers have difficulty in tuning suitable to load because of disturbance, parameter variation and noise.To overcome these difficulties conventional PID controllers were modified and developed lately by using various newer techniques. The controller development based on Fuzzy logic, neural network and Fuzzy-neural have been proposed as the better choice. However the Fuzzy logic controller (FLC) when compared to conventional controller, the main advantage of FLC is that no mathematical modeling required [3]. FLC controllers have excellent ability if a simple control algorithm is implemented. However this method has low reliability because the control results are sensitive to change in system parameters and do not react rapidly to parameter changes.

AC Servo systems are extensively used in robot actuator, machining tools, positioning control servomechanisms, computer numerical control and Industrial control [4]. AC Servo system is competing with DC servo system for motion control because of their favorable electrical and mechanical properties with good dynamic behavior with high efficiency.

AC servomotor is basically a two phase reversible induction motor and is capable of providing the desired response characteristics, due to their smaller diameter and high resistance rotors. The small diameter provides low inertia and helps for faster starts, stops and speed reversals while high resistance helps to shape the speed torque characteristics suitable for accurate control.

The evolving trend adopted by researchers for control systems infuses the use of machine learning or intelligence along with the conventional control techniques. Researchers have incorporated intelligence using techniques like neural networks [6][7], genetic algorithms [8] [17],evolutionary computing [9] and fuzzy logic [10][14]. The use neuro-fuzzy techniques for control is also gaining popularity [11][12] [13][15]. The use of neuro fuzzy systems is not adopted as the combination of such complex control systems possesses huge computational complexities for real time applications as considered by the authors of this paper.

This paper considers a direct torque control [DTC] of the asynchronous motors. A survey conducted has shown researchers adopt fuzzy logic [16] for the control but the results discussed in the paper lack accuracy of control. The use of neuro fuzzy logic proposed by researchers in [17] [18] demonstrated good learning capabilities, enabled to address the issue of a large torque ripple at 
low operating speeds but could not reduce the computational complexity of the overall system. The open research issues that exist are clearly discussed in [17] [18] [19].A comprehensive literature review of the techniques proposed by researcher's in the parameter control of motor drives is presented in [20]. This paper presented here adopts the use of neural networks for adaptive DTC of the ac servo motor.

\section{ARTIFICIAL NEURAL NETWORKS}

Some researchers have suggested that Artificial Neural Network (ANN) is the better choice for the controller design, since one could obtain superior and highly precise control response than a conventional PID controller. This is because the proposed controller can tune the conventional PID controller parameters more accurately with neural network technology since it requires only inputs and outputs even in Jacobian of unknown control objects. Also neural network are best in identifying patterns or trends in data, they are also better suited for prediction or Forecasting.

The learning ability, self adapting and fast computing futures of ANN make it well suited for medical diagnosis, medical research, voice recognition, machinery control, air traffic control and control of electrical power system in many application such as electric load forecasting, transient ability assessment, active power filter, dynamic voltage restorer, unified power quality conditioner etc. In learning processes neural network adjusts its structure such that it will be able to follow the supervisors set point. The learning is repeated until the difference between new output and the supervisor set point is low. Neural network based PID controller implementation and its computational task involved is so small that the on line adaptation becomes easy.

An artificial neural network is an information processing paradigm that is inspired by the way biological nervous system, such as brain process information. The key element of this model is the novel structure of the information processing system. It is composed of a large number of highly interconnected processing elements working in unison to solve specific problems. ANN is like where the people learn by examples [5]. Neural network take a different approach to problem solving than that of conventional computing techniques. Conventional computers use an algorithmic approach i.e., the computer follows a set of instructions in order to solve a problem that restricts the problem solving capability. But computers are more useful if they do things that we don't exactly know how to do it. Neural network processes information in a similar way the human brain does. The network is composed of a large number of highly inter connected processing elements (neurons) working in parallel to solve a specific problem. Neural network is a kind of continuous time dynamic system with high nonlinearity and good learning ability [6].

\section{MOTOR CONTROL}

In this section, speed control loop for a single phase asynchronous motor is demonstrated. The machine is fed by a PWM inverter. The speed control loop has a PID based regulator which produces a quadrature-axis current reference. The motor electromagnetic torque is controlled by this quadrature current. Hence, this method is also known as direct torque control (DTC). The motor flux is controlled by the direct-axis current. Transformation from $d-q$ frame to $a-b$ frame is used and the resulting currents are fed to the main and auxiliary motor windings.

The asynchronous motor has two windings: the main winding and the auxiliary winding. The electrical part of the machine is represented by a fourth order system whereas themechanical part is represented by a second order system. The electrical parameters are referred to the stator reference frame (d-q frame). The reference frame transformation ( $a-b$ to $d-q$ frame) is given by the following equation 1-2. The electrical behavior of the $\mathrm{AC}$ motor is modeled by equations $3-8$, while the mechanical behavior is modeled by equations $9-10$.

$$
\begin{gathered}
{\left[\begin{array}{l}
V_{q s} \\
V_{d s}
\end{array}\right]=\left[\begin{array}{cc}
1 & 0 \\
0 & -1
\end{array}\right]\left[\begin{array}{c}
V_{a s} \\
V_{b s}
\end{array}\right]} \\
{\left[\begin{array}{c}
V_{q r} \\
V_{d r}
\end{array}\right]=\left[\begin{array}{cc}
\cos \theta_{r} & -\sin \theta_{r} \\
-\sin \theta_{r} & \cos \theta_{r}
\end{array}\right]\left[\begin{array}{l}
V_{a r} \\
V_{b r}
\end{array}\right]}
\end{gathered}
$$

The flux linkage equations for the direct and the quadrature axes are given by equations (3)-(8).

$$
\begin{gathered}
\frac{d \Psi_{q s}^{s}}{d t}=w_{b}\left\{v_{q s}^{s}+\frac{r_{s}}{x_{l s}}\left(\Psi_{m q}^{s}-\Psi_{q s}^{s}\right)\right\} \\
\frac{d \Psi_{d s}^{s}}{d t}=w_{b}\left\{v_{d s}^{s}+\frac{r_{s}}{x_{l s}}\left(\Psi_{m d}^{s}-\Psi_{d s}^{s}\right)\right\} \\
\frac{d \Psi_{q r}^{s}}{d t}=w_{b}\left\{v_{q r}^{s}-\frac{w_{r}}{w_{b}} \Psi_{d r}^{s}+\frac{r_{r}}{x_{l r}}\left(\Psi_{m q}^{s}-\Psi_{q r}^{s}\right)\right\}(5) \\
\frac{d \Psi_{d r}^{s}}{d t}=w_{b}\left\{v_{d r}^{s}-\frac{w_{r}}{w_{b}} \Psi_{q r}^{s}+\frac{r_{r}}{x_{l r}}\left(\Psi_{m d}^{s}-\Psi_{d r}^{s}\right)\right\}(6) \\
\Psi_{m q}^{s}=x_{m}\left(i_{q s}^{s}+i_{q r}^{s}\right) \\
\Psi_{m d}^{s}=x_{m}\left(i_{d s}^{s}+i_{d r}^{s}\right)
\end{gathered}
$$

The electromechanical torque is given by

$T_{e m}=\frac{3}{2} \frac{\mathrm{P}}{2} \omega_{\mathrm{b}}\left(\psi_{\mathrm{ds}}^{\mathrm{s}} \mathrm{i}_{\mathrm{qs}}^{\mathrm{s}}-\psi_{\mathrm{qs}}^{\mathrm{s}} \mathrm{i}_{\mathrm{ds}}^{\mathrm{s}}\right)$

The dynamics of the rotor is given by equation

$$
J \frac{d w_{r m}}{d t}=T_{e m}+T_{\text {mec }}-T_{d a m p}
$$

Using MATLAB a SIMULINK model is used for system level simulations and modelling. The block diagram is as shown in Figure1. 


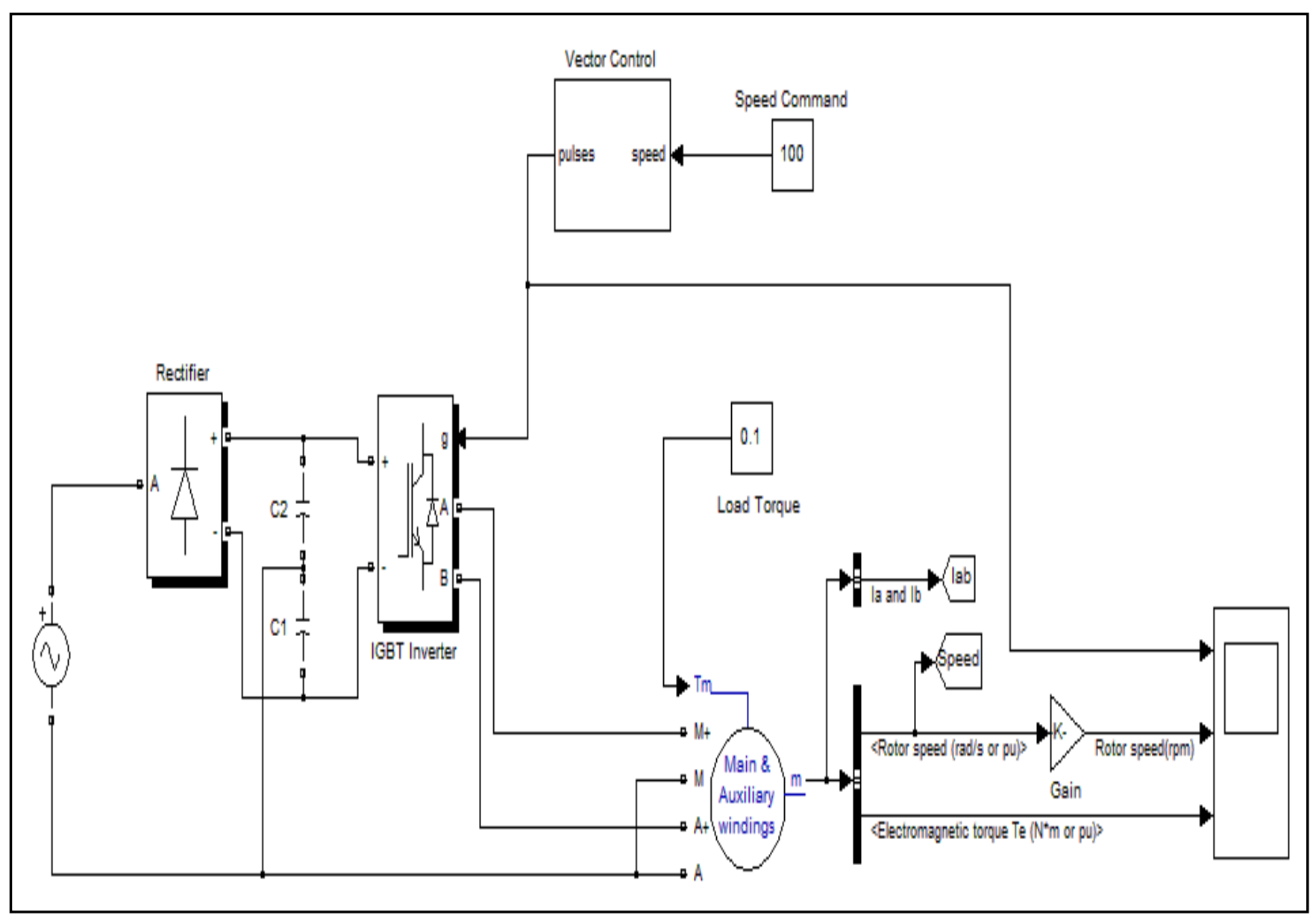

Fig1: SIMULINK Model for Motor Speed Control

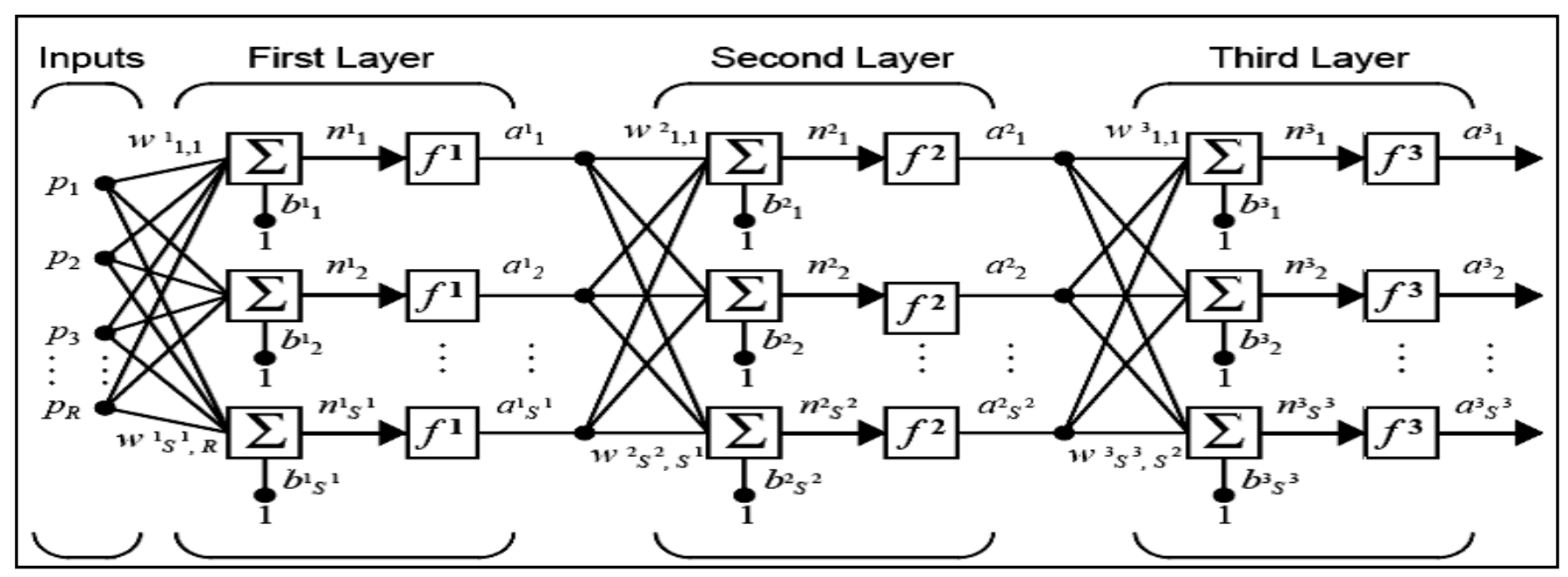

Fig2: Multi-layer neural network architecture

\section{NEURAL NETWORK ARCHITECTURE}

A multilayer neural network is shown in Fig 2. Each layer has its own weight matrix $\mathrm{W}$, its own bias vector $\mathbf{b}$, a net input vector $\mathbf{n}$ and an output vector a [6]. 
A layer whose output is the network output is called an output layer while other layers are called hidden layers. Multilayer networks are more powerful than single layer networks. In the present simulations, feed-forward neural network architecture is implemented in MATLAB. The neural network takes in the motor parameter variations and the dynamic load conditions as the input and generates the PID controller gains as the output. An input matrix with different input parameter values is fed to the neural network and the neural network is trained using the gradient descent back-propagation algorithm. Back-propagation is used to calculate derivatives of performance with respect to the weight and bias variables $\mathrm{X}$ and each variable is adjusted according to gradient descent:

$$
d X=\operatorname{lr} * \operatorname{dperf} / d X
$$

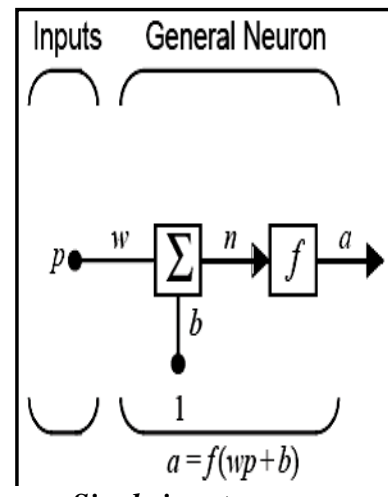

Single input neuron

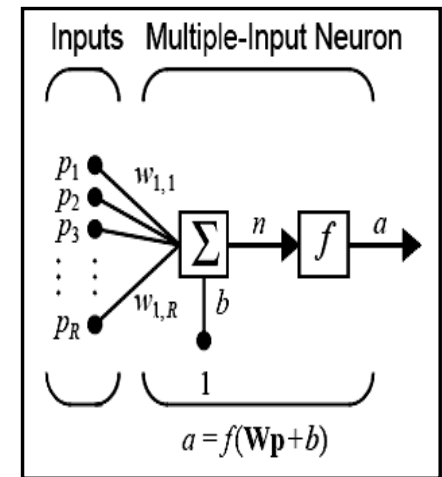

Multi-input neuron
Fig 3 Neuron models

MATLAB function newff( ) is used to create a feedforward back-propagation network. 2 hidden layers are created with 10 neurons in each layer. The transfer function for hidden layer neurons is tansig(),while purelin() is used for the output layer neurons. The transfer functions for the two are plotted in Fig4. train( )is used for the neural network training. The training function is traingd()which implements neural network training using gradient descent back-propagation algorithm.

Three different single output, multiple input neural networks are designed to be trained for tuning the proportional, integral and derivative gains of the PID controller based on the motor parameter variations. Each neural network is trained for the same set of training parameters using the gradient descent backpropagation algorithm. The advantage of training and tuning three different neural networks is that the mean square error parameter used as a performance metric is better minimized when three independent networks are tuned as compared to a single network with multiple outputs.

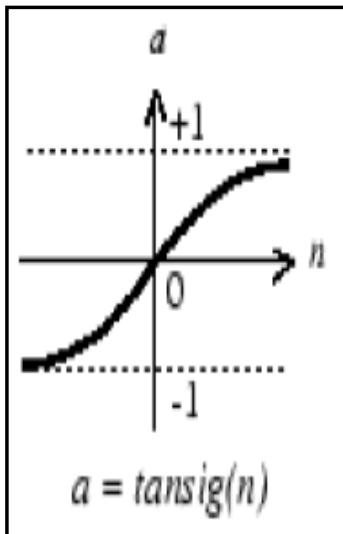

Hyperbolic tangent sigmoid function

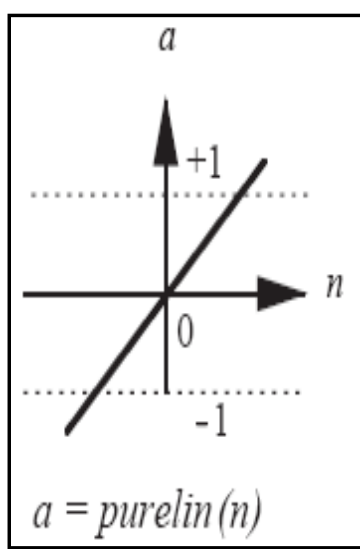

Pure Linear function
Fig 4 Neuron transfer functions

\section{RESULTS AND CONCLUSION}

A $0.25 \mathrm{HP}$ AC servo motor is used for the simulation model. The nominal motor parameters are listed in Table I.

TABLE I. Nominal motor parameters

\begin{tabular}{|c|c|}
\hline Parameter & Value \\
\hline Nominal Power & $0.25 \mathrm{HP}$ \\
\hline Stator resistance & $2.02 \mathrm{ohm}$ \\
\hline Stator inductance & $7.4 \mathrm{mH}$ \\
\hline Rotor resistance & $4.12 \mathrm{ohm}$ \\
\hline Rotor inductance & $5.6 \mathrm{mH}$ \\
\hline Inertia & $0.0146 \mathrm{kgm} 2$ \\
\hline
\end{tabular}

Fig 4 and 5 shows the speed control response of the motor for nominal motor parameters with different loads and friction coefficients. The speed command of $100 \mathrm{rpm}$ is tracked by the motor controller and the motor attains the commanded speed after some delay and slight overshoot.

Fig 6 and 7 shows the response of the motor with and without tuning of the PID gains using neural network, for smaller variations and for larger variations. It can be seen that the gradient descent back-propagation algorithm is able to tune the PID gains in-spite of the parameter variations. However the performance improvement is more for smaller variations as compared to larger variations. 


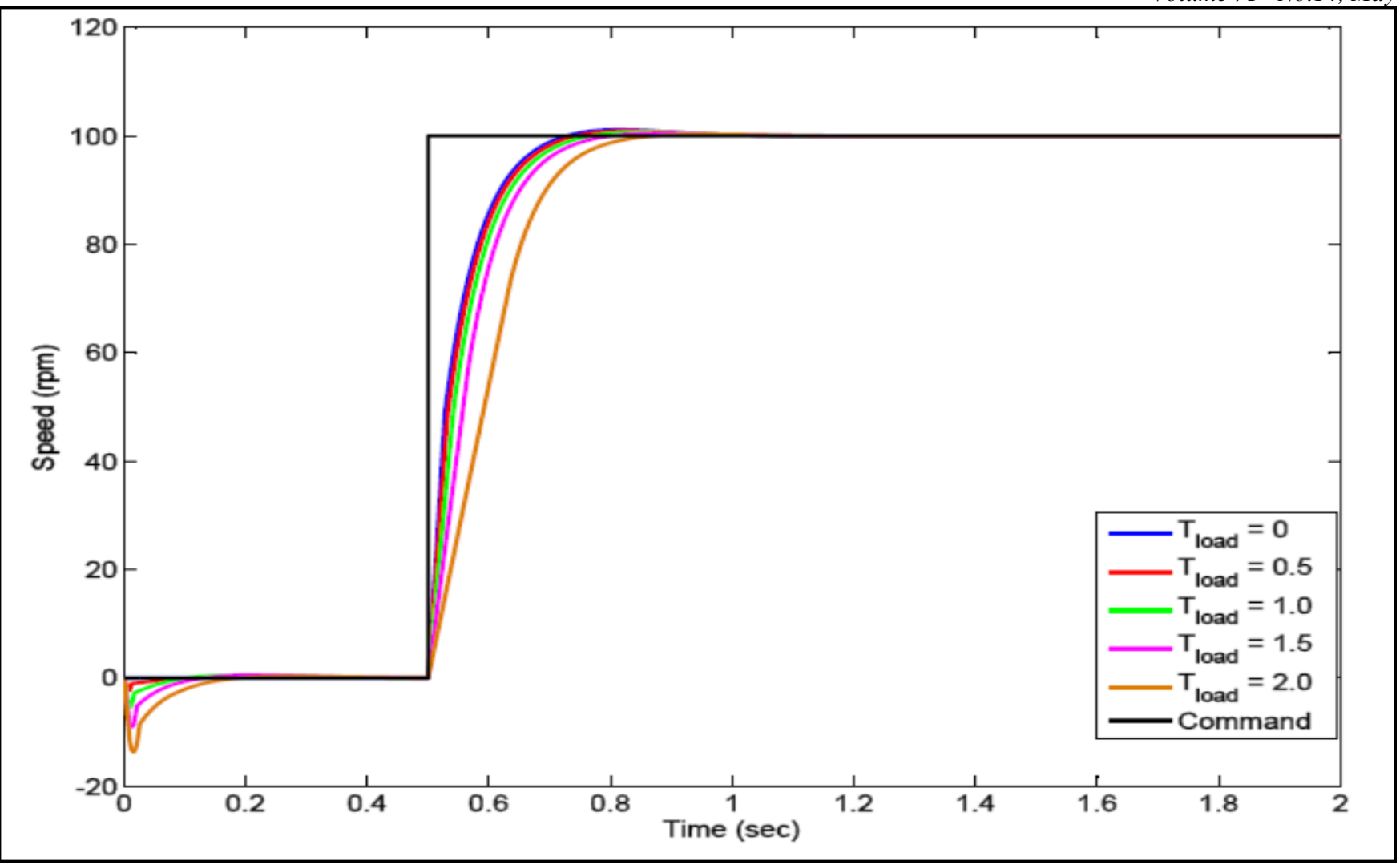

Fig 4: Nominal Speed Response for different loads

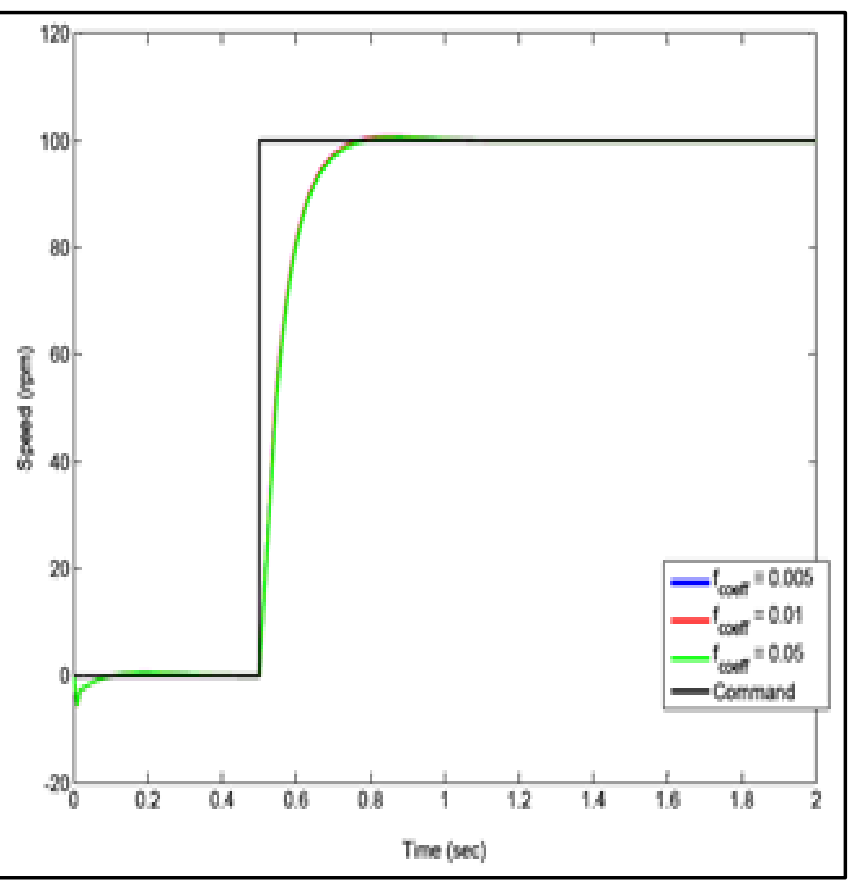

Fig 5: Speed Response for different friction coefficients

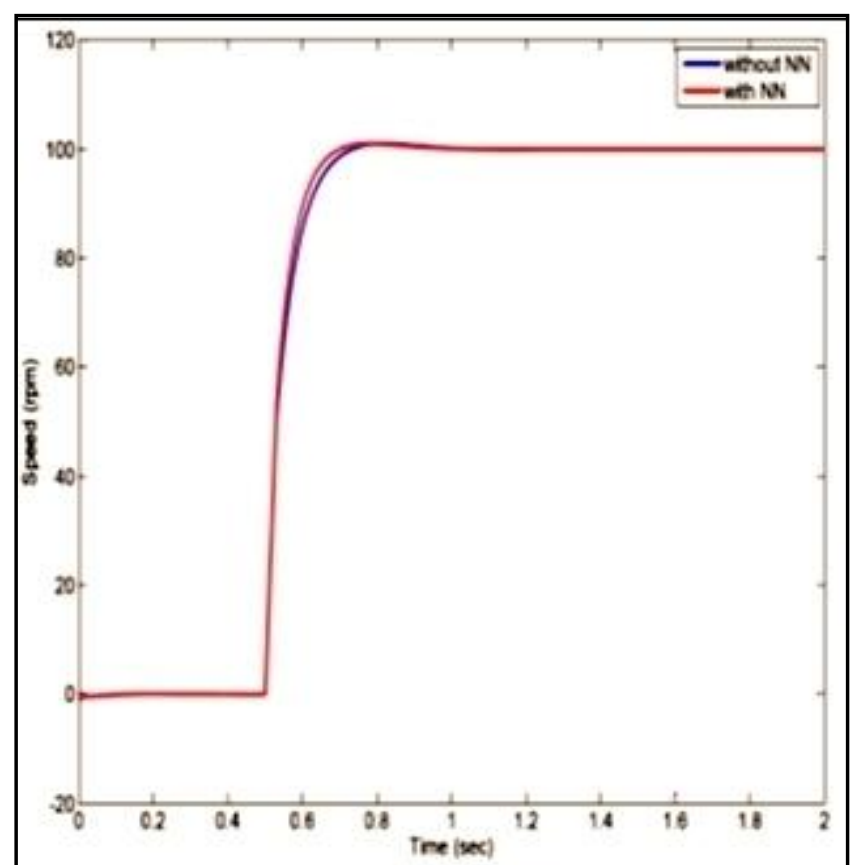

Fig 6: Comparison of response with and without neural network tuning (lesser parameter variations) 


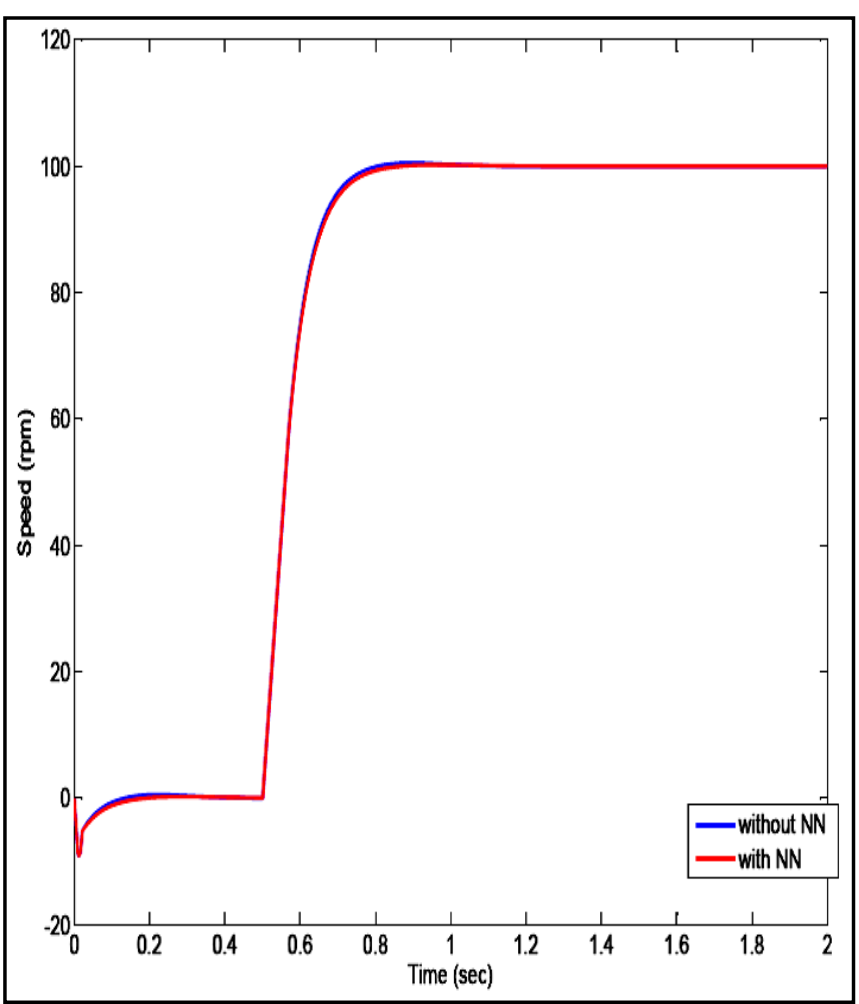

Fig 7: Comparison of response with and without neural network tuning (larger variations)

\section{ACKNOWLEDGMENT}

The authors would like to express their cordial thanks to MrAshutosh Kumar of Planet-i Technologies for their much valued support and advice.

\section{REFERENCES}

[1] A. Kaya and T. J. Scheib, "Tuning of PID controls of different structures," Control Eng., vol. 35, no. 7, pp. 62-65, Jul. 1988.

[2] Woo-Yoog Han, Sang-Min Kim, Sung-Joong Kim, ChangGoo Lee sensorless vector control of induction motor using improved self -tuning fuzzy PID controller. ICE: Annual conference in Fukui, August 4-6, 2003.

[3] Pyoung-Ho kim, Sa-Hyun sin, Hyung-Lae baek, Geum-Bae Cho, Speed control of AC servo motor neural networks.

[4] Principles of soft computing, S.N. Sivanandam, S.N. Deepa

[5] K.J. Hunt, D. Sbarbaro, R. Zbikowski, P.J. Gawthrop, "Neural Networks for Control Systems: A Survey, Automatica",pp. 1083-1122, 1992.

[6] Hagan, Martin T., Howard B. Demuth, and Mark H. Beale. Neural

Network design. Boston London: Pws Pub., 1996.

[7] A. M. Zou and K. D. Kumar, "Neural network-based distributed attitude coordination control for spacecraft formation flying with input saturation," IEEE Trans. Neural Netw. Learn. Syst., vol. 23, no. 7, pp. 1155-1162, Jul. 2012.
[8] M B Anandaraju, Dr. P S Puttaswamy and Jaswant Singh Rajpurohit. Article: Genetic Algorithm: An approach to Velocity Control of an Electric DC Motor. International Journal of Computer Applications 26(1):37-43, July 2011. Published by Foundation of Computer Science, New York, USA

[9] M B Anandaraju and P S Puttaswamy. Article: Modified Interactive Evolutionary Computing for Speed Control of an Electric DC Motor. International Journal of Computer Applications 39(15):19-24, February 2012. Published by Foundation of Computer Science, New York, USA

[10] M. Seera, C. P. Lim, D. Ishak, and H. Singh, "Fault detection and

[11] diagnosis of induction motors using motor current signature analysis and a hybrid FMM-CART model," IEEE Trans. Neural Netw. Learn. Syst., vol. 23, no. 1, pp. 97-108, Jan. 2012.

[12] R. J. Wai and P. C. Chen, "Intelligent tracking control for robot

[13] manipulator including actuator dynamics via TSK-type fuzzy neural network," IEEE Trans. Fuzzy Syst., vol. 12, no. 4, pp. 552-559, Aug. 2004

[14] R. J. Wai and Z. W. Yang, "Adaptive fuzzy neural network control design via a T-S fuzzy model for a robot manipulator including actuator dynamics," IEEE Trans. Syst., Man, Cybern. B, vol. 29, no. 5,pp. 583-591, Oct. 2008.

[15] Rong-Jong Wai, Rajkumar Muthusamy," Fuzzy-NeuralNetwork Inherited Sliding-Mode Control for Robot Manipulator Including Actuator Dynamics" IEEE Transactions On Neural Networks And Learning Systems, Vol. 24, No. 2, February 2013

[16] G. Feng, "A survey on analysis and design of model-based fuzzy control systems," IEEE Trans. Fuzzy Syst., vol. 14, no. 5, pp. 676-697, May 2006.

[17] R. Sharma and M. Gopal, "A Markov game adaptive fuzzy controller for robot manipulators," IEEE Trans. Fuzzy Syst., vol. 16, no. 1, pp. 171-186, Feb. 2008.

[18] L. ROMERAL, A. ARIAS, E. ALDABAS, "Novel direct torque control scheme with fuzzy adaptive torque ripple reduction". IEEE Trans on Industrial Electronics, vol. 50, pp. 487-492, 2003.

[19] Faa-Jeng Lin, Senior Member, IEEE, Po-Kai Huang, and Wen-Der Chou, "Recurrent-Fuzzy-Neural-NetworkControlled Linear Induction Motor Servo Drive Using Genetic Algorithms", IEEE Trans on Industrial Electronics, vol. 54, pp. 1449-1461, 2007.

[20] Shu Dasong . "A Direct Torque Control of Asynchronous Motor Based on Fuzzy Net-work", 2011 6th IEEE Conference on Industrial Electronics and Applications. pp 728-731. 2011

[21] Sun Xiao Hui, Zhang Zeng Ke, Han Ceng Jin. "Direct torque control of induction motor torque ripple minimization method", CSEE, vol. 22, pp. 109-112,2002.

[22] E. Levi, R. Bojoi, F. Profumo, H.A. Toliyat, S. Williamson. "Multiphase induction motor drives-a technology status review”, IET Electr. Power Appl, vol. 1, pp. 489-516, 2007. 